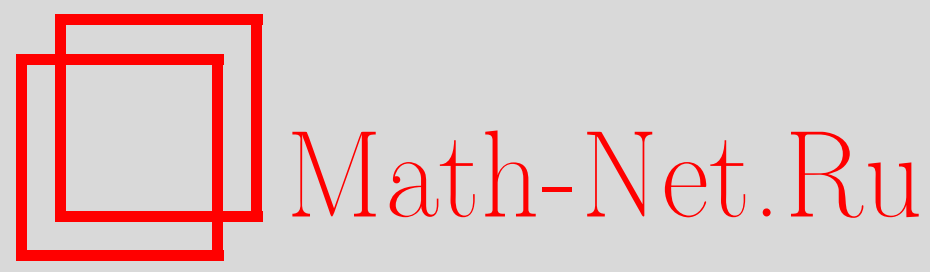

В. А. Емеличев, М. К. Кравцов, Д. П. Подкопаев, О квазиустойчивости траекторных задач векторной оптимизации, Матем. заметки, 1998, том 63, выпуск 1, 21-27

DOI: https://doi.org/10.4213/mzm1244

Использование Общероссийского математического портала Math-Net.Ru подразумевает, что вы прочитали и согласны с пользовательским соглашением http://www . mathnet.ru/rus/agreement

Параметры загрузки:

IP : 35.173 .219 .149

26 апреля 2023 г., 16:33:52

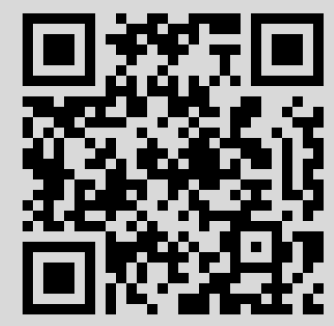




\title{
О КВАЗИУСТОЙЧИВОСТИ ТРАЕКТОРНЫХ ЗАДАЧ ВЕКТОРНОЙ ОПТИМИЗАЦИИ
}

\author{
В.А. Емеличев, М.К. Кравцов, Д. П. Подкопаев
}

\begin{abstract}
Выделен класс квазиустойчивых многокритериальных задач дискретной оптимизации на системах подмножеств (траекторных задач), которые при "малых" возмущениях (в чебьшевской метрике) коэффициентов целевых функций допускают возможность появления новых паретовских оптимумов при сохранении старых. В случае линейных критериев (MINSUM) выведена формула для вычисления радиуса квазиустойчивой задачи.

Библиография: 13 названий.
\end{abstract}

Признание проблемы устойчивости одной из центральных в математических исследованиях восходит к Ж. Адамару [1], которьй включил требование устойчивости в определение корректной, т.е. разумно поставленной задачи. Традиционно под устойчивостью задачи оптимизаиии (как однокритериальной, так и многокритериальной) понимается непрерывная зависимость решения от параметров задачи. Наиболее общие подходы к исследованию устойчивости оптимизационных задач основаны на изучении свойств многозначных (точечно-множественных) отображений, задающих функцию выбора (см., например, [2]-[5]).

Для исследования устойчивости задач дискретной оптимизации недостаточно традиционных методов математического анализа. В большей степени это объясняется сложностью дискретных моделей, которыепри незначительных изменениях в исходных данных часто ведут себя непредсказуемо [6], [7]. В то же время, отказ от использования общетопологической терминологии применительно к пространству изолированных точек значительно упрощает постановку проблемы устойчивости. В частности, свойство полунепрерьвности сверху (снизу) по Хаусдорфу оптимального отображения эквивалентно свойству непоявления новых решений (сохранения исходных решений) задачи при "малых" возмущениях ее параметров. В этом контексте возникает понятие устойчивости [6]-[12] (квазиустойчивости [6], [7], [11], [12]) задач дискретной оптимизации.

В настоящей заметке исследуется квазиустойчивость векторной траекторной задачи (т.е. задачи на системе подмножеств конечного множества) с наиболее распространенными в дискретной оптимизации частными критериями. Найдены нижняя достижимая оценка радиуса квазиустойчивости и критерий квазиустойчивости такой задачи. Получены также легко проверяемые достаточные условия квазиустойчивости.

Работа выполнена при финансовой поддержке Фонда фундаментальных исследований Республики Беларусь, грант $\Phi 95-70$. 
Ранее подобные исследования проводились для векторных задач целочисленного линейного программирования [12], а также для траекторных задач с линейньми критериями [11].

1. Определения. Рассмотрим систему подмножеств $(E, T)$, где $E=\left\{e_{1}, \ldots, e_{m}\right\}$, $T$ - некоторая совокупность непустых подмножеств множества $E$, назьваемых траекториями. Будем в дальнейшем предполагать, что $|T|>1$. Пусть на множестве $E$ задана векторная весовая функция $w(e)=\left(w_{1}(e), \ldots, w_{r}(e)\right) \in \mathbb{R}^{r}, r \geqslant 2$, а на множестве траекторий $T$ - векторньй критерий

$$
F(t)=\left(F_{1}(t), \ldots, F_{r}(t)\right), \quad F_{s}(t) \rightarrow \min _{T} \quad \forall s \in N_{r}=\{1,2, \ldots, r\},
$$

частными критериями которого могут быть критерии четырех видов, наиболее часто встречающихся в дискретной оптимизации:

$$
\begin{aligned}
\text { MINSUM: } F_{s}(t) & =\sum_{e \in t} w_{s}(e) \rightarrow \min _{T} ; \\
\text { MINMAX: } F_{s}(t) & =\max _{e \in t} w_{s}(e) \rightarrow \min _{T} ; \\
\text { MINMIN: } F_{s}(t) & =\min _{e \in t} w_{s}(e) \rightarrow \min _{T} ; \\
\text { MINPROD: } F_{s}(t) & =\prod_{e \in t} w_{s}(e) \rightarrow \min _{T}
\end{aligned}
$$

в любой комбинации. Будем обозначать через $I_{\mathrm{SUM}}, I_{\mathrm{MAX}}, I_{\mathrm{MIN}}, I_{\mathrm{PROD}}$ множества тех чисел из $N_{r}$, которыми занумерованы соответственно критерии MINSUM, MINMAX, MINMIN и MINPROD.

Под $r$-критериальной $(r \geqslant 2)$ траекторной задачей будем понимать задачу нахождения паретовского множества $P \subseteq T$, состоящего из всех паретовских оптимумов (эффективных траекторий) [4], [13]: $\widetilde{t} \in P$ тогда и только тогда, когда ни одна траектория из $T$ не доминирует траекторию $\widetilde{t}$, т.е. не существует траектории $t^{0} \in T$ такой, что $F(\widetilde{t}) \geqslant F\left(t^{0}\right), F(\widetilde{t}) \neq F\left(t^{0}\right)$.

Векторную весовую функцию $w(e)$ удобно представлять в виде матрицы $W=$ $\left\|w_{s k}\right\|_{r \times m}$, где $w_{s k}=w_{s}\left(e_{k}\right)$. Если предположить, что множества $E, T, I_{\mathrm{SUM}}, I_{\mathrm{MAX}}$, $I_{\mathrm{MIN}}$ и $I_{\mathrm{PROD}}$ зафиксированы, то матрица $W$ может служить для индексации $r$-критериальной задачи. В дальнейшем такую задачу будем обозначать через $Z^{r}(W)$, паретовское множество $P$ - через $P(W)$, значение $s$-го критерия $F_{s}(t)$ - через $F_{s}(t, W)$, а векторньй критерий $F(t)$ - через $F(t, W)$.

В схему траекторных задач вкладываются многие широко известные задачи оптимизации на графах, задачи булевого программирования и некоторые задачи теории расписаний (см., например, [8], [9]).

В пространстве $\mathbb{R}^{r m}$ зададим норму $l_{\infty}$, т.е. под нормой матрицы $B=\left\|b_{s k}\right\| \in \mathbb{R}^{r m}$ будем понимать число $\|B\|=\max \left\{\left|b_{s k}\right|:(s, k) \in N_{r} \times N_{m}\right\}$.

2. Квазиустойчивость. Пусть $\mathscr{B}(\varepsilon)=\left\{B \in \mathbb{R}^{r m}:\|B\|<\varepsilon\right\}, \varepsilon>0$. Задачу $Z^{r}(W+B)$, полученную из исходной задачи $Z^{r}(W)$ при сложении матрищ $W$ и $B \in \mathscr{B}(\varepsilon)$, будем назьвать возмущенной, а матрицу $B$ - возмущающей.

Задачу $Z^{r}(W)$ назовем квазиустойчивой, если существует число $\varepsilon>0$ такое, что выполняются включения

$$
P(W) \subseteq P(W+B) \quad \forall B \in \mathscr{B}(\varepsilon)
$$


Траектория $t^{*} \in T$ задачи $Z^{r}(W), r \geqslant 2$, называется [13] cтрого әффективной, если не сушествует траектории $t^{0} \in T, t^{*} \neq t^{0}$, такой, что $F\left(t^{*}, W\right) \geqslant F\left(t^{0}, W\right)$. Множество всех строго эффективных траекторий задачи $Z^{r}(W)$ будем обозначать через $S(W)$. Легко видеть, что $S(W) \subseteq P(W)$. Очевидна следующая

Лемма. $S(W)=P(W)$ тогда и только тогда, когда

$$
F\left(t_{1}, W\right) \neq F\left(t_{2}, W\right) \quad \forall t_{1}, t_{2} \in P(W), \quad t_{1} \neq t_{2} .
$$

ТеоРема 1. Если векторный критерий $F(t, W)$ представляет собой любую комбиначию критериев MINSUM, MINMAX, MINMIN $и$ MINPROD, то для того чтобъ задача $Z^{r}(W), r \geqslant 2$, бъла квазиустойчивой, достаточно, а в случае, когда векторный критерий содержит хотя бы один частный критерий вида MINSUM или MINPROD ( $\left.I_{\mathrm{SUM}} \cup I_{\mathrm{PROD}} \neq \varnothing\right)$, и необходимо, итобы выполнялось равенство $S(W)=P(W)$.

ДокАЗАТЕЛЬСтво. Достаточность. Пусть $S(W)=P(W)$. Тогда согласно лемме имеем $F\left(t_{1}, W\right) \neq F\left(t_{2}, W\right) \quad \forall t_{1}, t_{2} \in P(W), t_{1} \neq t_{2}$. Поэтому для любых траекторий $\widetilde{t} \in P(W), t^{0} \in T, \widetilde{t} \neq t^{0}$, существует индекс $s \in N_{r}$ такой, что $F_{s}\left(t^{0}, W\right)>F_{s}(\widetilde{t}, W)$. Значит, найдется число $\varepsilon>0$ такое, что $F_{s}\left(t^{0}, W+B\right)>F_{s}(\widetilde{t}, W+B) \forall B \in \mathscr{B}(\varepsilon)$. Следовательно, $\widetilde{t} \in P(W+B) \forall B \in \mathscr{B}(\varepsilon)$, т.е. задача квазиустойчива.

Доказательство необходимости проведем от противного. Пусть $S(W) \neq P(W)$. Тогда в силу леммы найдется пара различных эффективных траекторий $t_{1}, t_{2} \in P(W)$ такая, что $F\left(t_{1}, W\right)=F\left(t_{2}, W\right)$. Не ограничивая общности, можно предположить, что $t_{2} \backslash t_{1} \neq \varnothing$.

Пусть $\varepsilon$-произвольное положительное число. Рассмотрим два случая.

Случай 1 . $I_{\mathrm{SUM}} \neq \varnothing$. Пусть $p \in I_{\mathrm{SUM}}, e_{q} \in t_{2} \backslash t_{1}$. Рассмотрим возмущающую матрицу $C=C(\alpha)=\left\|c_{s k}\right\| \in \mathscr{B}(\varepsilon)$ с элементами

$$
c_{s k}= \begin{cases}\alpha, & \text { если }(s, k)=(p, q), \\ 0 & \text { для остальных }(s, k),\end{cases}
$$

где $0<\alpha<\varepsilon$. Тогда имеем

$$
F_{p}\left(t_{1}, W+C\right)<F_{p}\left(t_{2}, W+C\right), \quad F_{s}\left(t_{1}, W+C\right)=F_{s}\left(t_{2}, W+C\right) \quad \forall s \in N_{r}, \quad s \neq p .
$$

Поэтому $t_{2} \notin P(W+C)$. Следовательно, с учетом принадлежности $t_{2} \in P(W)$ заключаем, что задача $Z^{r}(W)$ не является квазиустойчивой.

Случай 2. $I_{\mathrm{PROD}} \neq \varnothing$. Пусть $p \in I_{\mathrm{PROD}}, e_{q} \in t_{2} \backslash t_{1}$.

Сначала предположим, что $F_{p}\left(t_{1}, W\right) \neq 0$. Тогда для матрицы $C=C(\alpha)($ см. $(2))$ имеем (3), т.е. $t_{1} \notin P(W+C)$ или $t_{2} \notin P(W+C)$. Следовательно, задача $Z^{r}(W)$ не является квазиустойчивой.

Пусть теперь $F_{p}\left(t_{1}, W\right)=0$. Положим $0<\beta<\varepsilon, B=\left\|b_{s k}\right\|_{r \times m}$, где

$$
b_{s k}= \begin{cases}\beta, & \text { если } s=p, w_{s k}=0, \\ 0 & \text { для остальных }(s, k) .\end{cases}
$$

Тогда $F_{p}\left(t_{1}, W+B\right) \neq 0$. 
Если $F_{p}\left(t_{1}, W+B\right) \neq F_{p}\left(t_{2}, W+B\right)$, то с учетом равенств

$$
F_{s}\left(t_{1}, W+B\right)=F_{s}\left(t_{2}, W+B\right) \quad \forall s \in N_{r}, \quad s \neq p
$$

получаем соотношение $t_{1} \notin P(W+B)$ или $t_{2} \notin P(W+B)$.

Если $F_{p}\left(t_{1}, W+B\right)=F_{p}\left(t_{2}, W+B\right)$, то $F_{p}\left(t_{1}, W+B+C\right) \neq F_{p}\left(t_{2}, W+B+C\right)$ при $C=C(\alpha), 0<\alpha<\varepsilon-\beta$. Поэтому с учетом равенств

$$
F_{s}\left(t_{1}, W+B+C\right)=F_{s}\left(t_{2}, W+B+C\right) \quad \forall s \in N_{r}, \quad s \neq p
$$

имеем $t_{1} \notin P(W+B+C)$ или $t_{2} \notin P(W+B+C)$.

Таким образом, с учетом принадлежностей $B \in \mathscr{B}(\varepsilon), B+C \in \mathscr{B}(\varepsilon)$ заключаем, что задача $Z^{r}(W)$ не является квазиустойчивой.

Собирая все доказанное, убеждаемся в справедливости теоремы 1.

Приведем пример, показьвающий, что в случае, когда $I_{\mathrm{SUM}} \cup I_{\mathrm{PROD}}=\varnothing$, достаточное условие теоремы 1 не является необходимым.

Пусть $m=3, r=2, T=\left\{t_{1}, t_{2}\right\}, t_{1}=\left\{e_{1}, e_{2}\right\}, t_{2}=\left\{e_{2}, e_{3}\right\}$,

$$
W=\left(\begin{array}{lll}
1 & 3 & 2 \\
3 & 1 & 2
\end{array}\right), \quad F_{1}(t, W)=\max _{e \in t} w_{1}(e), \quad F_{2}(t, W)=\min _{e \in t} w_{2}(e) .
$$

Тогда $F\left(t_{1}, W\right)=F\left(t_{2}, W\right)=(3,1)$. Поэтому $P(W)=T, S(W)=\varnothing$.

Тем не менее, задача $Z^{2}(W)$ квазиустойчива, поскольку при $\varepsilon=1 / 2$ выполняются включения (1).

ЗАмЕчАниЕ 1. В работе [12] было доказано, что равенство $S(W)=P(W)$ является необходимьм и достаточньм условием квазиустойчивости многокритериальной задачи целочисленного линейного программирования, а в [11] получен критерий квазиустойчивости траекторной задачи $Z^{r}(W)$ в случае, когда $N_{r}=I_{\mathrm{SUM}}$.

Пусть $W\left(I_{\mathrm{SUM}}\right)$ - подматрица матрицы $W$, состоящая из строк матрицы $W$ с номерами $I_{\mathrm{SUM}}$.

СлЕДСТВИЕ 1. Задача $Z^{r}(W), W=\left\|w_{s k}\right\|_{r \times m}, r \geqslant 2$, квазиустойчива, если $\left|I_{\mathrm{SUM}}\right| \geqslant m u \operatorname{rank} W\left(I_{\mathrm{SUM}}\right)=m$.

ДокАЗАТЕЛЬСТво. Прежде всего заметим, что всякую функцию $F_{s}(t, W), s \in I_{\mathrm{SUM}}$, можно представить в виде скалярного произведения векторов $w_{s}=\left(w_{s} 1, \ldots, w_{s m}\right)$ и $x(t)=\left(x_{1}, \ldots, x_{m}\right)$, где

$$
x_{i}=\left\{\begin{array}{l}
1, \text { если } e_{i} \in t, \\
0, \text { если } e_{i} \notin t,
\end{array} \quad i \in N_{m}\right.
$$

Очевидно, что

$$
x\left(t_{1}\right) \neq x\left(t_{2}\right) \quad \forall t_{1}, t_{2} \in T, \quad t_{1} \neq t_{2} .
$$

Пусть задача $Z^{r}(W)$ не является квазиустойчивой. Тогда согласно лемме и теореме 1 существуют две различные траектории $t_{1}, t_{2} \in P(W)$ такие, что выполняется равенство $W\left(I_{\mathrm{SUM}}\right)\left(x\left(t_{1}\right)-x\left(t_{2}\right)\right)^{T}=\mathbf{0}$, где $\mathbf{0}=(0, \ldots, 0)^{T} \in \mathbb{R}^{q}, q=\left|I_{\mathrm{SUM}}\right|$. Однако, это равенство противоречит условиям следствия и соотношениям (4). Следствие доказано. 
ТЕоремА 2. Задача $Z^{r}(W), r \geqslant 2$, квазиустойчива, если векторный критерий представляет собой любую комбиначию критериев вида MINMIN и MINMAX $\left(I_{\mathrm{SUM}} \cup I_{\mathrm{PROD}}=\varnothing\right)$, а в каждой строке матрииы $W$ әлементы попарно различны.

ДокАЗАТЕЛьство. Для доказательства теоремы 2 достаточно показать, что для каждой пары различных траекторий $\widetilde{t} \in P(W), t^{0} \in T$ найдется число $\varepsilon>0$, удовлетворяющее следующему условию.

УСловиЕ А. Траектория $t^{0}$ не доминирует траекторию $\widetilde{t}$ ни в какой возмущенной задаче $Z^{r}(W+B) \forall B \in \mathscr{B}(\varepsilon)$.

Случай 1. $F(\widetilde{t}, W) \neq F\left(t^{0}, W\right)$. Тогда для некоторого индекса $s \in N_{r}$ будет вьполняться неравенство $F_{s}(\widetilde{t}, W)<F_{s}\left(t^{0}, W\right)$. Следовательно, найдется достаточно малое положительное число $\varepsilon$, удовлетворяющее указанному вьше условию А.

Случай 2. $F(\widetilde{t}, W)=F\left(t^{0}, W\right)$. Так как каждый критерий имеет вид MINMAX или MINMIN, то для всякого индекса $s \in N_{r}$ и всякой траектории $t \in T$ существует индекс $k=k(s, t)$ такой, что $F_{s}(t, W)=w_{s k}$. Тем самьг, равенство $F(\widetilde{t}, W)=F\left(t^{0}, W\right)$ преврашается в равенства $w_{s k(s, \widetilde{t})}=w_{s k\left(s, t^{0}\right)} \forall s \in N_{r}$. А поскольку элементы каждой строки матрицы $W$ попарно различны, то $k(s, \widetilde{t})=k\left(s, t^{0}\right)=k(s) \forall s \in N_{r}$. Следовательно,

$$
F_{s}(\widetilde{t}, W)=F_{s}\left(t^{0}, W\right)=w_{s k(s)} \quad \forall s \in N_{r}
$$

Пусть

$$
\varepsilon=\frac{1}{3} \min _{s \in N_{r}} \min _{1 \leqslant i<j \leqslant m}\left|w_{s i}-w_{s j}\right| .
$$

Тогда с учетом (5) для любой возмущающей матрицы $B \in \mathscr{B}(\varepsilon)$ имеем

$$
F_{s}(\widetilde{t}, W+B)=F_{s}\left(t^{0}, W+B\right)=w_{s k(s)}+b_{s k(s)} \quad \forall s \in N_{r}
$$

Таким образом, $F(\widetilde{t}, W+B)=F\left(t^{0}, W+B\right) \forall B \in \mathscr{B}(\varepsilon)$. Следовательно, условие А выполняется. Теорема доказана.

ЗАмЕчАнИЕ 2. В однокритериальной задаче $Z^{1}(W)$ (матрица $W$ превращается в вектор-строку) аналогом паретовского множества $P(W)$ является множество всех оптимальных траекторий. Легко сформулировать однокритериальные аналоги теорем 1 и 2.

ЗАмечАнИЕ 3. С учетом эквивалентности любых двух норм в конечномерном линейном пространстве теоремы 1,2 и следствие 1 справедливы и для других норм в пространстве возмущающих матриц $\mathbb{R}^{r m}$.

3. Радиус квазиустойчивости. Радиусом квазиустойчивости задачи $Z^{r}(W)$, $r \geqslant 2$, по аналогии с радиусом устойчивости (см. [11]) назовем число $\rho(W)=\sup \{\varepsilon$ : $P(W) \subseteq P(W+B) \forall B \in \mathscr{B}(\varepsilon)\}$.

Введем обозначение

$$
\Phi(W)=\min _{\tilde{t} \in P(W)} \min _{t \in T \backslash\{\widetilde{t}\}} \max _{s \in N_{r}} \Gamma_{s}^{W}(t, \widetilde{t}),
$$


где

$$
\begin{aligned}
\Gamma_{s}^{W}\left(t_{1}, t_{2}\right) & =\frac{\tau_{s}^{W}\left(t_{1}, t_{2}\right)}{\Delta_{s}\left(t_{1}, t_{2}\right)}, \quad \tau_{s}^{W}\left(t_{1}, t_{2}\right)=F_{s}\left(t_{1}, W\right)-F_{s}\left(t_{2}, W\right), \\
\Delta_{s}\left(t_{1}, t_{2}\right) & = \begin{cases}\left|t_{1}\right|+\left|t_{2}\right|-2\left|t_{1} \cap t_{2}\right|, & \text { если } s \in I_{\mathrm{SUM}}, \\
2, & \text { если } s \in I_{\mathrm{MIN}} \cup I_{\mathrm{MAX}} .\end{cases}
\end{aligned}
$$

Теорема 3. Для радиуса квазиустойчивости задачи $Z^{r}(W), r \geqslant 2, c$ любой комбинацией критериев MINSUM, MINMAX $и$ MINMIN $\left(I_{\mathrm{PROD}}=\varnothing\right)$ справедлива оченка $\rho(W) \geqslant \Phi(W)$.

ДокАЗАтЕльство. Если $\Phi(W)=0$, то утверждение теоремы очевидно. Если $\Phi(W) \geqslant \varepsilon>0$, то для любых двух траекторий $\widetilde{t} \in P(W), t^{0} \in T, \widetilde{t} \neq t^{0}$, вьполняется неравенство $\max \left\{\Gamma_{s}^{W}\left(t^{0}, \widetilde{t}\right): s \in N_{r}\right\} \geqslant \varepsilon$. Покажем, что существует индекс $p \in N_{r}$ такой, что $\tau_{p}^{W+B}\left(t^{0}, \widetilde{t}\right)>0 \quad \forall B \in \mathscr{B}(\varepsilon)$.

Пусть $p \in \operatorname{argmax}\left\{\Gamma_{s}^{W}\left(t^{0}, \widetilde{t}\right): s \in N_{r}\right\}$. Тогда имеем

$$
\Gamma_{p}^{W}\left(t^{0}, \widetilde{t}\right) \geqslant \varepsilon>\|B\| \quad \forall B \in \mathscr{B}(\varepsilon) .
$$

Поэтому получаем

$$
\begin{aligned}
\tau_{p}^{W+B}\left(t^{0}, \widetilde{t}\right) & \geqslant \tau_{p}^{W}\left(t^{0}, \widetilde{t}\right)-\Delta_{p}\left(t^{0}, \widetilde{t}\right) \max \left\{\left|b_{p j}\right|: j \in N_{m}\right\} \\
& \geqslant \tau_{p}^{W}\left(t^{0}, \widetilde{t}\right)-\|B\| \Delta_{p}\left(t^{0}, \widetilde{t}\right)>0 \quad \forall B \in \mathscr{B}(\varepsilon)
\end{aligned}
$$

Отсюда вьводим

$$
P(W) \subseteq P(W+B) \quad \forall B \in \mathscr{B}(\varepsilon) .
$$

Следовательно, $\rho(W) \geqslant \Phi(W)$. Теорема доказана.

СлЕДСТВИЕ 2. Если векторный критерий задачи $Z^{r}(W), r \geqslant 2$, состоит лишь из критериев вида MINSUM $\left(I_{\mathrm{SUM}}=N_{r}\right)$, mо

$$
\rho(W)=\Phi(W) .
$$

ДокАЗАтЕльство. Пусть $\varepsilon>\Phi(W)$. Тогданайдутся траектории $\widetilde{t} \in P(W), t^{0} \in T$, $\widetilde{t} \neq t^{0}$, такие, что $\gamma=\max \left\{\Gamma_{s}^{W}\left(t^{0}, \widetilde{t}\right): s \in N_{r}\right\}<\varepsilon$. Покажем, что существует возмущающая матрица $C \in \mathscr{B}(\varepsilon)$, для которой $\tau_{s}^{W+C}\left(t^{0}, \widetilde{t}\right)<0 \forall s \in N_{r}$.

Рассмотрим матрицу $C \in \mathscr{B}(\varepsilon)$, устроенную следующим образом. Пусть $\gamma<c<\varepsilon$. Если $\widehat{t}=t^{0} \backslash \widetilde{t} \neq \varnothing$, то

$$
c_{s k}=\left\{\begin{array}{r}
-c \text { при } e_{k} \in \widehat{t}, s \in N_{r}, \\
c \text { в противном случае. }
\end{array}\right.
$$

Если $\widehat{t}=\varnothing$, то $c_{s k}=c \forall(s, k) \in N_{r} \times N_{m}$.

Тогда с учетом линейности частных критериев $\left(I_{\mathrm{SUM}}=N_{r}\right)$ получаем

$$
\begin{aligned}
\tau_{s}^{W+C}\left(t^{0}, \widetilde{t}\right) & =\tau_{s}^{W}\left(t^{0}, \widetilde{t}\right)+F_{s}\left(t^{0}, C\right)-F_{s}(\widetilde{t}, C)=\tau_{s}^{W}\left(t^{0}, \widetilde{t}\right)-\|C\| \Delta_{s}\left(t^{0}, \widetilde{t}\right) \\
& <\Delta_{s}\left(t^{0}, \widetilde{t}\right)\left(\Gamma_{s}^{W}\left(t^{0}, \widetilde{t}\right)-\gamma\right) \leqslant 0 \quad \forall s \in N_{r} .
\end{aligned}
$$

Это означает, что для возмущающей матрицы $C$ справедливо соотношение $P(W) \not \subset$ $P(W+C)$. Следовательно, $\rho(W) \leqslant \Phi(W)$, и с учетом теоремы 3 следствие доказано.

ЗАМЕчАНИЕ 4 . Нетрудно видеть, что формула (6) справедлива и при $r=1$. 


\section{СПИСОК ЦИТИРОВАННОЙ ЛИТЕРАТУРЫ}

[1] Hadamard J. Sur les problèmes aux derivées partielles et leur signification partielle et leur signification physique. Bulletin: Princeton Univ., 1902.

[2] Молодцов Д. А., Федоров В. В. Устойчивость принципов оптимальности // Современное состояние теории исследования операций. М.: Наука, 1979. С. 236-263.

[3] Е. Г. Белоусов, Б. Банк (ред. ) Математическая оптимизация: вопросы разрешимости и устойчивости. М.: Изд-во МГУ, 1986.

[4] Дубов Ю.А., Травкин С. И., Якимец В. Н. Многокритериальные модели формирования и выбора вариантов систем. М.: Наука, 1986.

[5] Молодцов Д. А. Устойчивость принципов оптимальности. М.: Наука, 1987.

[6] Козерацкая Л.Н., Лебедева Т. Т., Сергиенко И. В. Исследование устойчивости задач дискретной оптимизации // Кибернетика и системный анализ. 1993. №3. С. 78-93.

[7] Сергиенко И. В., Козерацкая Л.Н., Лебедева Т. Т. Исследование устойчивости и параметрический анализ дискретных оптимизационных задач. Киев: Навукова думка, 1995.

[8] Леонтьев В.К. Устойчивость задачи коммивояжера // ЖВМиМФ. 1975. Т. 15. № 5 . C. $1298-1309$.

[9] Sotskov Yu. N., Leontev V. K., Gordeev E. N. Some concepts of stability analysis in combinatorial optimization // Discrete Appl. Math. 1995. V. 58. P. 169-190.

[10] Гордеев Э. Н., Леонтьев В. К. Общий подход к исследованию устойчивости решений в задачах дискретной оптимизации // ЖВМиМФ. 1996. Т. 36. №1. С. 66-72.

[11] Емеличев В.А., Кравцов М.К. Об устойчивости в траекторных задачах векторной оптимизации // Кибернетика и системный анализ. 1995. № 4. С. 137-143.

[12] Козерацкая Л.Н., Лебедева Т. Т., Сергиенко Т. И. Задачи целочисленного программирования с векторным критерием: параметрический анализ и исследование устойчивости // Докл. АН СССР. 1989. Т. 307. № 3. С. 527-529.

[13] Подиновский В. В., Ногин В. Д. Парето-оптимальные решения многокритериальных задач. М.: Наука, 1982. 